\title{
A new structure of an integral operator associated with trigonometric Dunkl settings
}

Shrideh Khalaf Al-Omari ${ }^{1 *}$ (D), Serkan Araci $^{2}$ and Mohammed Al-Smadi ${ }^{3,4}$

"Correspondence:

s.k.q.alomari@fet.edu.jo;

shridehalomari@bau.edu.jo

${ }^{1}$ Department of Physics and Basic

Sciences, Faculty of Engineering

Technology, Al-Balqa Applied

University, 11134, Amman, Jordan

Full list of author information is

available at the end of the article

\section{Springer}

\begin{abstract}
In this paper, we discuss a generalization to the Cherednik-Opdam integral operator to an abstract space of Boehmians. We introduce sets of Boehmians and establish delta sequences and certain class of convolution products. Then we prove that the extended Cherednik-Opdam integral operator is linear, bijective and continuous with respect to the convergence of the generalized spaces of Boehmians. Moreover, we derive embeddings and discuss properties of the generalized theory. Moreover, we obtain an inversion formula and provide several results.
\end{abstract}

MSC: Primary 54C40; 14E20; secondary 46E25; 20 C20

Keywords: Cherednik-Opdam integral operator; Convolution product; Polynomial; Differential-difference operator; Boehmian

\section{Introduction and preliminaries}

Generalized functions were designed by Sobolev and Schwartz to fulfil the apparent requirements of science. Being an extension to the concept of ordinary functions, the theory of generalized functions gives rise to many fruitful results in partial differential equations, yet at the same time they are arbitrarily singular. In literature, the notion of generalized functions have witnessed a volcanic growth in PDEs, physics, engineering, mathematical physics, theoretical stochastic analysis and some numerical aspects as well. Typically generalized functions are defined as continuous linear mappings on appropriately defined spaces of test functions, nevertheless, Boehmians are introduced as quotients of convolution products similar to the concept of field of quotients (see, e.g., [1-5] and [6-10]). Although the construction of a Boehmian space might be obtained by convolution products and delta sequences of shrinking supports to the origin, it may not be possible to define a notion of a Boehmian when the delta sequences fail to vanish on an open set. The idea of such a construction has led to many important ideas on the support of a Boehmian and the abelian-type theorems of the integral transform operators.

Here and throughout, it being understood conventionally that $\mathbb{C}, \mathbb{R}$ and $\mathbb{N}$ are the sets of complex numbers, real numbers and positive integers, respectively. For arbitrary but fixed real parameters $\alpha$ and $\beta$ subject to the constraints $\alpha \geq \beta \geq-1 / 2, \alpha>-1 / 2$ and $\lambda \in \mathbb{C}$, the Opdam-Cherednik theory is a theory based on the Opdam-Cherednik normalized eigen

(c) The Author(s) 2021. This article is licensed under a Creative Commons Attribution 4.0 International License, which permits use, sharing, adaptation, distribution and reproduction in any medium or format, as long as you give appropriate credit to the original author(s) and the source, provide a link to the Creative Commons licence, and indicate if changes were made. The images or other third party material in this article are included in the article's Creative Commons licence, unless indicated otherwise in a credit line to the material. If material is not included in the article's Creative Commons licence and your intended use is not permitted by statutory regulation or exceeds the permitted use, you will need to obtain permission directly from the copyright holder. To view a copy of this licence, visit http://creativecommons.org/licenses/by/4.0/. 
function $G_{\lambda}^{(\alpha, \beta)}$ of the Dunkl-Cherednik differential-difference operator $F^{(\alpha, \beta)}$, where

$$
F^{(\alpha, \beta)} G_{\lambda}^{(\alpha, \beta)}(t)=i \lambda G_{\lambda}^{(\alpha, \beta)}(t)
$$

provided $G_{\lambda}^{(\alpha, \beta)}(0)=1, F^{(\alpha, \beta)} f(t)=f^{\prime}(t)+\{(\alpha-\beta) \operatorname{coth} t+(2 \beta+1) \operatorname{coth} 2 t\} F(t)-p f(-t)$, $p=\alpha+\beta+1$ and $F(t)=f(t)-f(-t)$ (see, e.g., [11]). An efficient relation between the hypergeometric and the Jacobi functions, $G_{\lambda}^{(\alpha, \beta)}$ and $\varphi_{\lambda}^{(\alpha, \beta)}$, was established as

$$
G_{\lambda}^{(\alpha, \beta)}(t)=\varphi_{\lambda}^{(\alpha, \beta)}(t)-\frac{1}{p-i \lambda} \frac{\partial}{\partial t} \varphi_{\lambda}^{(\alpha, \beta)}(t)
$$

where $\varphi_{\lambda}^{(\alpha, \beta)}(t)={ }_{2} F_{1}\left(\frac{p+i \lambda}{2}, \frac{p-i \lambda}{2} ; \alpha+1 ;-\sinh ^{2} t\right),{ }_{2} F_{1}$ being the hypergeometric function. A translation formula, in this division, was introduced as

$$
\tau_{x}^{(\alpha, \beta)} f(y)=\int_{\mathbb{R}} f(w) d \mu_{x, y}^{(\alpha, \beta)}(w),
$$

where

$$
\begin{aligned}
& d \mu_{x, y}^{(\alpha, \beta)}(w)= \begin{cases}K_{\alpha, \beta}(x, y, w) A_{\alpha, \beta}(w) d w, & x y \neq 0, \\
d \delta_{x}(w), & y=0, \\
d \delta_{y}(w), & x=0,\end{cases} \\
& K_{\alpha, \beta}(x, y, w)=A_{x y w} \int_{0}^{-2 \alpha} g_{x, y, w, \theta}^{\alpha-\beta-1}\left(1-\sigma_{x, y, w}^{\theta}+\sigma_{x, w, y}^{\theta}+\sigma_{w, y, x}^{\theta}+h_{x, y, w, \theta}^{\beta, \alpha}\right) \sin ^{2 \beta} \theta d \theta, \\
& A_{x y, w}=\frac{\mu_{\alpha+\beta}}{|\sinh x \sinh y \sinh w|^{2 \alpha},} \quad h_{x, y, w, \theta}^{\beta, p}=\frac{\alpha \operatorname{coth} x \operatorname{coth} y \operatorname{coth} w \sin ^{2} \theta}{\beta+\frac{1}{2}}, \\
& x, y, w \in \mathbb{R} \backslash\{0\}, \quad \text { and } \\
& g_{x, y, w, \theta}=1-\cosh ^{2} x-\cosh ^{2} y \cosh ^{2} w+2 \cosh x \cosh y \cosh w \cos \theta,
\end{aligned}
$$

satisfy the triangular inequality ||$x|-| y||<|w|<|x|+|y|$, provided that

$$
\sigma_{x, y, w}^{\theta}= \begin{cases}\frac{\cosh x+\cosh y-\cosh w \cos \theta}{\sinh w \sinh y}, & x y \neq 0, \\ 0, & x y=0,\end{cases}
$$

for $x, y, w \in \mathbb{R}, \theta \in[0,1]$ and $K_{\alpha, \beta}(x, y, w)=0$ otherwise. It will be very useful to report here that a change of variables in $K_{\alpha, \beta}$, for $x, y, w \in \mathbb{R}$, yields (see, e.g., [12])

$$
K_{\alpha, \beta}(x, y, w)=K_{\alpha, \beta}(y, x, w)=K_{\alpha, \beta}(-x, w, y)=K_{\alpha, \beta}(-w, y,-x) .
$$

In the literature, the Opdam-Cherednik integral operator is defined, on the space $C_{c}(\mathbb{R})$ of continuous functions with compact support on $\mathbb{R}$, as a Fourier integral operator in trigonometric Dunkl settings given by [13]

$$
\gamma(f)(\lambda)=\int_{\mathbb{R}} f(t) G_{\lambda}^{(\alpha, \beta)}(-t) \vec{d}_{\alpha, \beta} t,
$$


where $\alpha \geq \beta \geq-\frac{1}{2}, \alpha>-\frac{1}{2}$ and $\lambda \in \mathbb{C}$. The inversion formula of the Opdam-Cherednik integral operator can be recovered from the Opdam-Cherednik integral operator as

$$
\gamma^{-1}(h)(\lambda)=\int_{\mathbb{R}} h(\lambda) G_{\lambda}^{(\alpha, \beta)}(t)\left(1-\frac{p}{i \lambda}\right) \frac{1}{8 \pi\left|C_{\alpha, \beta}(\lambda)\right|^{2}} d \lambda .
$$

The Roe and the Paley-Wiener theorems in the context of Cherednik operators were established by using tempered distributions with spectral gaps in the Opdam-Cherednik operator (see, e.g., $[14,15]$ ). On the other hand, uncertainty principles and local uncertainty principles of Donoho-Strak type were derived by Achak and Daher [16]. Further, in an attractive perspective, they have adequately established certain analogs of Hardy, Beurling, Cowling-Price, Gelfand-Shilov and Miyachi theorems, with the aid of composition properties of the Opdam-Cherednik operator. However, various investigations and real applications of this integral operator may be observed in $[11,12,17-20]$ and the references therein.

Although there were discussed various integral operators on different spaces of Boehmians, the theory of the Cherednik-Opdam integral operator of a Boehmian has not yet been reported in the literature. The starting point in such an approach relies on a convolution theorem which, in addition to delta sequences, allows embeddings to act as isomorphisms between the classical spaces $L^{1}\left(\mathbb{R}, \vec{d}_{\alpha, \beta} x\right)$ and $L_{E}^{1}\left(\mathbb{R}, \vec{d}_{\alpha, \beta} x\right), \vec{d}_{\alpha, \beta} x=A_{\alpha, \beta}(|x|) d x, A_{\alpha, \beta}(|x|)=$ $\sinh ^{2 \alpha+1}|x| \cosh ^{2 \beta+1}|x|$, and the generalized spaces $\beta_{1}^{(\alpha, \beta)}$ and $\beta_{2}^{(\alpha, \beta)}$ of Boehmians, respectively. Here and hereafter, we will be concerned with themes in the context of Boehmian spaces and the framework of the Cherednik-Opdam integral operator on the real line. We will consider a Cherednik-Opdam set of delta sequences and provide various axioms to legitimate the Cherednik-Opdam sets of Boehmians. Similar argument is also applied to the Cherednik-Opdam inversion formula. To be more precise, we expand our results into three sections. In Sect. 1, we recall the general description of the Opdam hypergeometric functions, convolution products and some related results. In Sect. 2, we define delta sequences and prove numerous results that presumably exhibits the Cherednik-Opdam spaces of Boehmians. In Sect. 3, we discuss the generalized theory of the CherednikOpdam integral and its inversion formula in the class of Boehmians.

\section{Opdam-Cherednik generalized spaces}

In this section, we generate two imperative sets of Boehmians and extend the OpdamCherednik integral operator to the given sets. For certain deterministic needs, we introduce Opdam-Cherednik sets of delta sequences, convolution products, convolution theorems and establish vital axioms for such extension. By $L^{1}\left(\mathbb{R}, \vec{d}_{\alpha, \beta} x\right)$, we denote the set of all measurable functions such that the integral formula

$$
\|f\|_{\alpha, \beta}=\int_{\mathbb{R}}|f(x)| \vec{d}_{\alpha, \beta} x
$$

is finite. Likewise, by $D(\mathbb{R})$ we denote the subspace of the measurable space $L^{1}\left(\mathbb{R}, \vec{d}_{\alpha, \beta} x\right)$ of test functions of compact supports over $\mathbb{R}$. The convolution product between two suitable functions $f_{1}$ and $f_{2}$ in the space $L^{1}\left(\mathbb{R}, \vec{d}_{\alpha, \beta} x\right)$ is defined, when the integral exists, as [13]

$$
f_{1} *_{\alpha, \beta} f_{2}(x)=\int_{\mathbb{R}} \tau_{x}^{(\alpha, \beta)} f_{1}(-y) f_{2}(y) \vec{d}_{\alpha, \beta} y .
$$


In the course of the following two lemmas, we recite some properties of the product $*_{\alpha, \beta}$ as follows.

Lemma 1 ([13, Remark 4.8]) Let $f_{1}, f_{2}, f_{3} \in L^{1}\left(\mathbb{R}, \vec{d}_{\alpha, \beta} x\right)$. Then the following holds:

$$
f_{1} *_{\alpha, \beta} f_{2}=f_{2} *_{\alpha, \beta} f_{1} \quad \text { and } \quad\left(f_{1} *_{\alpha, \beta} f_{2}\right) *_{\alpha, \beta} f_{3}=f_{1} *_{\alpha, \beta}\left(f_{2} *_{\alpha, \beta} f_{3}\right) .
$$

For $p=q=r=1$, the following result is very beneficial for the sequel.

Lemma 2 ([13, Proposition 4.10]) Let $f_{1}, f_{2} \in L^{1}\left(\mathbb{R}, \vec{d}_{\alpha, \beta} x\right)$. Then $f_{1} *_{\alpha, \beta} f_{2} \in L^{1}\left(\mathbb{R}, \vec{d}_{\alpha, \beta} x\right)$ and

$$
\left\|f_{1} *_{\alpha, \beta} f_{2}\right\|_{\alpha, \beta} \leq C_{\alpha, \beta}\left\|f_{1}\right\|_{\alpha, \beta}\left\|f_{2}\right\|_{\alpha, \beta},
$$

where the coefficients are given by

$$
C_{\alpha, \beta}= \begin{cases}4+\frac{\Gamma(\alpha+1) \Gamma\left(\beta+\frac{1}{2}\right)}{\Gamma\left(\alpha+\frac{1}{2}\right) \Gamma(\beta+1)}, & \alpha>\beta>-\frac{1}{2}, \\ \frac{5}{2}, & \alpha=\beta>-\frac{1}{2} .\end{cases}
$$

As delta sequences are essential parts in this treatment, the Opdam-Cherednik set of delta sequences can be presented as follows.

Definition 3 By $\Delta^{(\alpha, \beta)}$, we denote the subset of $D(\mathbb{R})$ consisting of all sequences $\left(\delta_{n}\right)$ such that the identities $P_{1}, P_{2}$ and $P_{3}$ hold:

$$
\begin{aligned}
& P_{1}: \int_{\mathbb{R}} \delta_{n}(x) G_{0}^{(\alpha, \beta)}(-x) \vec{d}_{\alpha, \beta} x=1 \quad \text { for every } n \in \mathbb{N}, \\
& P_{2}:\left\|\delta_{n}\right\|_{\alpha, \beta} \leq M \quad \text { for some constant } M>0 \text { and every } n \in \mathbb{N}, \\
& P_{3}: \lim _{n \rightarrow \infty} \int_{|x|>\epsilon}\left|\delta_{n}(x)\right| \vec{d}_{\alpha, \beta} x=0 \text { for every real number } \epsilon>0 .
\end{aligned}
$$

We have the following assertion.

Proposition 4 The set $\Delta^{(\alpha, \beta)}$ is an Opdam-Cherednik set of delta sequences.

Proof To show that $\Delta^{(\alpha, \beta)}$ is an Opdam-Cherednik set of delta sequences we have to show that $P_{1}-P_{3}$ hold for all $\Delta^{(\alpha, \beta)}$ elements. Let $\left(\delta_{n}\right),\left(\theta_{n}\right) \in \Delta^{(\alpha, \beta)}$. Then, by the convolution theorem $\gamma\left(f *_{\alpha, \beta} g\right)(\lambda)=\gamma(f)(\lambda) \gamma(g)(\lambda)$ which can deduced from [13, Proposition 4.9] and [21], we write

$$
\gamma\left(\delta_{n} *_{\alpha, \beta} \theta_{n}\right)(0)=\gamma\left(\delta_{n}\right)(0) \gamma\left(\theta_{n}\right)(0) .
$$

Therefore, we rewrite (7) in an explicit form as

$$
\int_{\mathbb{R}}\left(\delta_{n} *_{\alpha, \beta} \theta_{n}\right)(x) G_{0}^{\alpha, \beta}(-x) \vec{d}_{\alpha, \beta} x=\int_{R} \delta_{n}(x) G_{0}^{\alpha, \beta}(-x) \vec{d}_{\alpha, \beta} x \int_{\mathbb{R}} \theta_{n}(x) G_{0}^{\alpha, \beta}(-x) \vec{d}_{\alpha, \beta} x
$$


Hence, from the previous equation it can be easily inferred that

$$
\int_{\mathbb{R}}\left(\delta_{n} *_{\alpha, \beta} \theta_{n}\right)(x) G_{0}^{\alpha, \beta}(-x) \vec{d}_{\alpha, \beta} x=1
$$

Hence, $P_{1}$ is completely proved. To prove $P_{2}$, let $\left(\delta_{n}\right)$ and $\left(\theta_{n}\right)$ be in $\Delta^{(\alpha, \beta)}$. Then we have $\left\|\delta_{n}\right\|_{\alpha, \beta} \leq M_{1}$ and $\left\|\theta_{n}\right\|_{\alpha, \beta} \leq M_{2}$ for some real numbers $M_{1}$ and $M_{2}$. Therefore, from Lemma 2, we get $\left\|\delta_{n} *_{\alpha, \beta} \theta_{n}\right\|_{\alpha, \beta} \leq M$ where $M=C_{\alpha, \beta} M_{1} M_{2}$. Finally, if $\lim _{n \rightarrow \infty} \int_{|x|>\epsilon}\left|\delta_{n}(x)\right| \vec{d}_{\alpha, \beta} x=0$ and $\lim _{n \rightarrow \infty} \int_{|x|>\epsilon}\left|\theta_{n}(x)\right| \vec{d}_{\alpha, \beta} x=0$, then we have

$$
\lim _{n \rightarrow \infty} \int_{|x|>\epsilon}\left|\left(\delta_{n} *_{\alpha, \beta} \theta_{n}\right)(x)\right| \vec{d}_{\alpha, \beta} x \leq \lim _{n \rightarrow \infty} \int_{|x|>\epsilon}\left|\delta_{n}(x)\right| \vec{d}_{\alpha, \beta} x \lim _{n \rightarrow \infty} \int_{|x|>\epsilon}\left|\theta_{n}(x)\right| \vec{d}_{\alpha, \beta} x .
$$

Hence, we have obtained

$$
\lim _{n \rightarrow \infty} \int_{|x|>\epsilon}\left|\left(\delta_{n} *_{\alpha, \beta} \theta_{n}\right)(x)\right| \vec{d}_{\alpha, \beta} x=0 .
$$

This completes the proof of the proposition.

We now establish the prerequisite axioms for generating the Boehmian space $\beta_{1}^{(\alpha, \beta)}$ with the set $L^{1}\left(\mathbb{R}, \vec{d}_{\alpha, \beta} x\right)$, the subset $D(\mathbb{R})$, the product $*_{\alpha, \beta}$ and the set $\Delta^{(\alpha, \beta)}$ of delta sequences.

Theorem 5 Let $f, g, h, f_{n} \rightarrow f$ as $n \rightarrow \infty$ in $L^{1}\left(\mathbb{R}, \vec{d}_{\alpha, \beta} x\right), \delta, \theta \in D(\mathbb{R})$ and $\alpha \in \mathbb{C}$. Then we have:

(i) $\alpha\left(f *_{\alpha, \beta} g\right)=(\alpha f) *_{\alpha, \beta} g$.

(ii) $(f+g) *_{\alpha, \beta} \delta=f *_{\alpha, \beta} \delta+g *_{\alpha, \beta} \delta$.

(iii) $f *_{\alpha, \beta} g=g *_{\alpha, \beta} f$, and $\left(f *_{\alpha, \beta} g\right) *_{\alpha, \beta} h=f *_{\alpha, \beta}\left(g *_{\alpha, \beta} h\right)$.

(iv) $f_{n} *_{\alpha, \beta} \delta \rightarrow f *_{\alpha, \beta} \delta$ as $n \rightarrow \infty$.

Proof The proofs of (i) and (ii) are straightforward. The proof of (iii) is consistent with the proof of Lemma 2. Hence, the proofs are omitted. The proof of (iv) follows from Lemma 2 and the fact that

$$
\left\|\left(f_{n}-f\right) *_{\alpha, \beta} \delta\right\|_{\alpha, \beta} \leq C_{\alpha, \beta}\left\|f_{n}-f\right\|_{\alpha, \beta}\|\delta\|_{\alpha, \beta} \rightarrow 0 \quad \text { as } n \rightarrow \infty
$$

This finishes the proof of the theorem.

Finally, we establish the following lemma.

Lemma 6 Let $f \in L^{1}\left(\mathbb{R}, \vec{d}_{\alpha, \beta} x\right)$ and $\left(\delta_{n}\right) \in \Delta^{(\alpha, \beta)}$. Then $f *_{\alpha, \beta} \delta_{n} \rightarrow f$ as $n \rightarrow \infty$ in $L^{1}\left(\mathbb{R}, \vec{d}_{\alpha, \beta} x\right)$.

Proof Let $\left(\delta_{n}\right) \in \Delta^{(\alpha, \beta)}$ and $f \in L^{1}\left(\mathbb{R}, \vec{d}_{\alpha, \beta} x\right)$ be given. Then by [11, Lemma 4.6] we have

$$
\left\|\tau_{x}^{(\alpha, \beta)} f\right\|_{\alpha, \beta} \leq C_{\alpha, \beta}\|f\|_{\alpha, \beta}
$$


where $C_{\alpha, \beta}$ has a significance of (6). Also, from [12] we see that the function $G_{0}^{(\alpha, \beta)}$ is strictly positive and bounded above and, for every $\lambda \in \mathbb{R}$, we have

$$
\left|G_{\lambda}^{(\alpha, \beta)}(x)\right| \leq G_{0}(x) \quad \text { for every } x \in \mathbb{R}
$$

Therefore, by Definition 3 and Fubini's theorem, we write

$$
\begin{aligned}
\| f & *_{\alpha, \beta} \delta_{n}-f \|_{\alpha, \beta} \\
& \leq \int_{\mathbb{R}}\left(\int_{\mathbb{R}}\left|\left(\tau_{x}^{(\alpha, \beta)} f(-y)-f(x) G_{0}^{(\alpha, \beta)}(-y)\right) \delta_{n}(y) \vec{d}_{\alpha, \beta} y\right|\right) \vec{d}_{\alpha, \beta} x \\
& \leq \int_{\mathbb{R}}\left(\int_{\mathbb{R}}\left|\tau_{x}^{(\alpha, \beta)} f(-y)-f(x) G_{0}^{(\alpha, \beta)}(-y)\right|\left|\delta_{n}(y)\right|\left|\vec{d}_{\alpha, \beta} y\right|\right) \vec{d}_{\alpha, \beta} x \\
& \leq \int_{\mathbb{R}}\left(\int_{\mathbb{R}}\left(\left|\tau_{x}^{(\alpha, \beta)} f(-y)\right|+\left|f(x) G_{0}^{(\alpha, \beta)}(-y)\right|\right)\left|\delta_{n}(y)\right|\left|\vec{d}_{\alpha, \beta} x\right|\right) \vec{d}_{\alpha, \beta} x .
\end{aligned}
$$

Let $C$ be an upper bound for $G_{0}^{(\alpha, \beta)}$, then, by [15, Proposition 4.4], we have

$$
\begin{aligned}
\| f & *_{\alpha, \beta} \delta_{n}-f \|_{\alpha, \beta} \\
& \leq \int_{\mathbb{R}}\left(\int_{\mathbb{R}}\left(\left|\tau_{y}^{(\alpha, \beta)} f(-x)\right|+C|f(x)|\right)\left|\delta_{n}(y)\right|\left|\vec{d}_{\alpha, \beta} y\right|\right) \vec{d}_{\alpha, \beta} x \\
& \leq \int_{\mathbb{R}}\left(C_{\alpha, \beta}\|f\|_{\alpha, \beta}+C\|f\|_{\alpha, \beta}\right)\left|\delta_{n}(y)\right|\left|\vec{d}_{\alpha, \beta} y\right| \\
& \leq\left(C_{\alpha, \beta}\|f\|_{\alpha, \beta}+C\|f\|_{\alpha, \beta}\right) \int_{\mathbb{R}}\left|\delta_{n}(y)\right|\left|\vec{d}_{\alpha, \beta} y\right| \\
& \leq\left(C_{\alpha, \beta}\|f\|_{\alpha, \beta}+C\|f\|_{\alpha, \beta}\right)\left\|\delta_{n}\right\|_{\alpha, \beta} \rightarrow 0 \text { as } n \rightarrow \infty .
\end{aligned}
$$

This finishes the proof of the theorem.

If $\left(\varphi_{n}\right) \in L^{1}\left(\mathbb{R}, \vec{d}_{\alpha, \beta} x\right)$ and $\left(\delta_{n}\right) \in \Delta^{(\alpha, \beta)}$, then the pair $\left(\varphi_{n}, \delta_{n}\right)$ (or $\left.\frac{\varphi_{n}}{\delta_{n}}\right)$ is said to be a quotient of sequences if $\varphi_{n} *_{\alpha, \beta} \delta_{m}=\varphi_{m} *_{\alpha, \beta} \delta_{n}, \forall n, m \in \mathbb{N}$. Therefore, if $\frac{\varphi_{n}}{\delta_{n}}$ and $\frac{g_{n}}{\varepsilon_{n}}$ are quotients of sequences and $\psi \in L^{1}\left(\mathbb{R}, \vec{d}_{\alpha, \beta} x\right)$, then it is easy to see that

$$
\frac{\psi *_{\alpha, \beta} \delta_{n}}{\delta_{n}} \text { and } \frac{\varphi_{n} *_{\alpha, \beta} \delta_{n}+g_{n} *_{\alpha, \beta} \delta_{n}}{\delta_{n} *_{\alpha, \beta} \varepsilon_{n}}
$$

are quotients of sequences. Further, we can easily check the following equivalence relations:

$$
\frac{\varphi_{n}}{\delta_{n} *_{\alpha, \beta} \psi} \sim \frac{\varphi_{n} *_{\alpha, \beta} \psi}{\delta_{n}} \text { and } \frac{\varphi_{n}}{\delta_{n} *_{\alpha, \beta} g_{n}} \sim \frac{\varphi_{n} *_{\alpha, \beta} g_{n}}{\delta_{n}} .
$$

Two quotients of sequences $\frac{\varphi_{n}}{\delta_{n}}$ and $\frac{g_{n}}{\varepsilon_{n}}$ are said to be equivalent if $\varphi_{n} *_{\alpha, \beta} \varepsilon_{m}=g_{m} *_{\alpha, \beta}$ $\delta_{n}, \forall n, m \in \mathbb{N}$. The equivalent class $\breve{w}=\left(\frac{\varphi_{n}}{\delta_{n}}\right)$ of quotients of sequences containing $\frac{\varphi_{n}}{\delta_{n}}$ is said to be a Boehmian. The space of such Boehmians is denoted by $\beta_{1}^{(\alpha, \beta)}$. For two Boehmians $\breve{w}=\left(\frac{\varphi_{n}}{\delta_{n}}\right)$ and $\breve{z}=\left(\frac{g_{n}}{\varepsilon_{n}}\right)$ in $\beta_{1}^{(\alpha, \beta)}$, the following are well-defined on $\beta_{1}^{(\alpha, \beta)}$ :
(i) $\breve{w}+\breve{z}=\left(\frac{\varphi_{n} *_{\alpha, \beta} \delta_{n}+g_{n} *_{\alpha, \beta} \delta_{n}}{\delta_{n} *_{\alpha, \beta} \varepsilon_{n}}\right)$,
(ii) $\beta \breve{w}=\left(\frac{\beta \varphi_{n}}{\delta_{n}}\right)$, 

(iii) $\breve{w} *_{\alpha, \beta} \breve{z}=\left(\frac{\varphi_{n} *_{\alpha, \beta} g_{n}}{\delta_{n} *_{\alpha, \beta} \varepsilon_{n}}\right)$
(iv) $D^{k} \breve{w}=\left(\frac{D^{k} \varphi_{n}}{\delta_{n}}\right)$, and
(v) $\breve{w} *_{\alpha, \beta} \psi=\left(\frac{\varphi_{n} *_{\alpha, \beta} \psi}{\delta_{n}}\right)$

where $k \in \mathbb{R}, \beta \in \mathbb{C}$ and $D^{k} \breve{w}$ is the $k$ th derivative of $\breve{w}$ and $\psi \in L^{1}\left(\mathbb{R}, \vec{d}_{\alpha, \beta} x\right)$.

Definition 7 For $n=1,2,3, \ldots$ and $\breve{w}_{n}, \breve{w} \in \beta_{1}^{(\alpha, \beta)}$, the sequence $\left(\breve{w}_{n}\right)$ is said to be $\delta$ convergent to $\breve{w}$, denoted by $\delta-\lim _{n \rightarrow \infty} \breve{w}_{n}=\breve{w}$, provided there can be found a delta sequence $\left(\delta_{n}\right)$ such that

(a) $\left(\breve{w}_{n} *_{\alpha, \beta} \delta_{k}\right),\left(\breve{w} *_{\alpha, \beta} \delta_{k}\right) \quad$ in $L^{1}\left(\mathbb{R}, \vec{d}_{\alpha, \beta} x\right)$, for all $n, k \in \mathbb{N}$,

(b) $\lim _{n \rightarrow \infty} \breve{w}_{n} *_{\alpha, \beta} \delta_{k}=\breve{w} *_{\alpha, \beta} \delta_{k} \quad$ in $L^{1}\left(\mathbb{R}, \vec{d}_{\alpha, \beta} x\right)$, for every $k \in \mathbb{N}$.

Definition 8 For $n=1,2,3, \ldots$ and $\breve{w}_{n}, \breve{w} \in \beta_{1}^{(\alpha, \beta)}$, the sequence $\left(\breve{w}_{n}\right)$ is said to be $\Delta^{(\alpha, \beta)}$ convergent to $\breve{w}$, denoted by $\Delta^{\alpha, \beta}-\lim _{n \rightarrow \infty} \breve{w}_{n}=\breve{w}$, provided there can be found a delta sequence $\left(\delta_{n}\right)$ such that
(a) $\left(\breve{w}_{n}-\breve{w}\right) *_{\alpha, \beta} \delta_{n} \in L^{1}\left(\mathbb{R}, \vec{d}_{\alpha, \beta} x\right) \quad(\forall n \in \mathbb{N})$,
(b) $\lim _{n \rightarrow \infty}\left(\breve{w}_{n}-\breve{w}\right) *_{\alpha, \beta} \delta_{n}=0 \quad$ in $L^{1}\left(\mathbb{R}, \vec{d}_{\alpha, \beta} x\right)$.

Remark 9 Let $\psi \in L^{1}\left(\mathbb{R}, \vec{d}_{\alpha, \beta} x\right)$ and $\left(\delta_{n}\right) \in \Delta^{(\alpha, \beta)}$ be fixed. Then we have the mapping

$$
\psi \rightarrow \breve{w}
$$

where $\breve{w}=\left(\frac{\psi * \alpha, \beta}{\delta_{n}}\right)$ is an injective mapping from $L^{1}\left(\mathbb{R}, \vec{d}_{\alpha, \beta} x\right)$ into $\beta_{1}^{(\alpha, \beta)}$.

Therefore, it can be easily checked that $L^{1}\left(\mathbb{R}, \vec{d}_{\alpha, \beta} x\right)$ may be identified as a subspace of $\beta_{1}^{(\alpha, \beta)}$.

Remark 10 Let $\left(\delta_{n}\right) \in \Delta^{(\alpha, \beta)}$. Then, if $\psi_{n} \rightarrow \psi$ in $L^{1}\left(\mathbb{R}, \vec{d}_{\alpha, \beta} x\right)$ as $n \rightarrow \infty$, then, for all $k \in \mathbb{N}$,

$$
\psi_{n} *_{\alpha, \beta} \delta_{k} \rightarrow \psi *_{\alpha, \beta} \delta_{k}
$$

as $n \rightarrow \infty$. That is, $\breve{w}_{n} \rightarrow \breve{w}$ in $\beta_{1}^{(\alpha, \beta)}$ as $n \rightarrow \infty$.

The above remark states the following.

Theorem 11 The mappings $\psi \rightarrow \breve{w}, \breve{w}=\left(\frac{\psi *_{\alpha, \beta} \delta_{n}}{\delta_{n}}\right)$, is a continuous embedding of the space $L^{1}\left(\mathbb{R}, \vec{d}_{\alpha, \beta} x\right)$ into the space $\beta_{1}^{(\alpha, \beta)}$.

Now, let $L_{E}^{1}\left(\mathbb{R}, \vec{d}_{\alpha, \beta} x\right), D_{E}(\mathbb{R})$ and $\Delta_{E}^{(\alpha, \beta)}$ be the Opdam-Cherednic operators of the spaces $L^{1}\left(\mathbb{R}, \vec{d}_{\alpha, \beta} x\right), D(\mathbb{R})$ and $\Delta^{(\alpha, \beta)}$, respectively. Then we have the following definition.

Definition 12 Let $F \in L_{E}^{1}\left(\mathbb{R}, \vec{d}_{\alpha, \beta} x\right)$ and $\Psi \in D_{E}(\mathbb{R})$. Then between $F$ and $\Psi$, we define an operation $*_{\alpha, \beta}^{E}$ as

$$
F *_{\alpha, \beta}^{E} \Psi(x)=(F \Psi)(x)
$$

where $(F \Psi)(x)=F(x) \Psi(x)$ is the usual product of two functions. 
Accordingly, the construction of the Boehmian space $\beta_{2}^{(\alpha, \beta)}$ with the sets $L_{E}^{1}\left(\mathbb{R}, \vec{d}_{\alpha, \beta} x\right)$, $D_{E}(\mathbb{R})$ and $\Delta_{E}^{(\alpha, \beta)}$ follows from the following theorem.

Theorem 13 Let $F, G, F_{n}, F$ be in $L_{E}^{1}\left(\mathbb{R}, \vec{d}_{\alpha, \beta} x\right), \psi_{1}, \psi_{2} \in D_{E}(\mathbb{R}),\left(\theta_{n}^{E}\right),\left(\delta_{n}^{E}\right) \in \Delta_{E}^{(\alpha, \beta)}, \bar{\alpha} \in \mathbb{C}$ and $F_{n} \rightarrow F$ as $n \rightarrow \infty$. Then we have

(i) $\bar{\alpha}\left(F *_{\alpha, \beta}^{E} G\right)=(\bar{\alpha} F) *_{\alpha, \beta}^{E} G$.

(ii) $\left(\theta_{n}^{E} *_{\alpha, \beta}^{E} \delta_{n}^{E}\right) \in \Delta_{E}^{(\alpha, \beta)}$.

(iii) $F *_{\alpha, \beta}^{E}\left(\psi_{1} *_{\alpha, \beta}^{E} \psi_{2}\right)=\left(F *_{\alpha, \beta}^{E} \psi_{1}\right) *_{\alpha, \beta}^{E} \psi_{2}$.

(iv) $F_{n} *_{\alpha, \beta}^{E} \psi_{1} \rightarrow F *_{\alpha, \beta}^{E} \psi_{1}$ as $n \rightarrow \infty$.

(v) $F *_{\alpha, \beta}^{E} \delta_{n}^{E} \rightarrow F$ as $n \rightarrow \infty$ in $L_{E}^{1}\left(\mathbb{R}, \vec{d}_{\alpha, \beta} x\right)$.

(vi) $(F+G) *_{\alpha, \beta}^{E} \psi_{1}=F *_{\alpha, \beta}^{E}+G *_{\alpha, \beta}^{E} \psi_{1}$.

Proof of (i) By using (12), two times, we get $\bar{\alpha}\left(F *_{\alpha, \beta}^{E} G\right)(\lambda)=\bar{\alpha}(F G)(\lambda)=(\bar{\alpha} F(\lambda)) G(\lambda)=$ $(\bar{\alpha} F) *_{\alpha, \beta}^{E} G$. This proves (i). To prove (ii), let $\left(\theta_{n}\right),\left(\delta_{n}\right) \in \Delta^{(\alpha, \beta)}$ be such that $\theta_{n}^{E}=\gamma \theta_{n}$ and $\delta_{n}^{E}=\gamma \delta_{n}$ for all $n \in \mathbb{N}$. Then, by [13, Proposition 4.9], we have

$$
\left(\theta_{n}^{E} *_{\alpha, \beta}^{E} \delta_{n}^{E}\right)(\lambda)=\left(\gamma \theta_{n} *_{\alpha, \beta}^{E} \gamma \delta_{n}\right)(\lambda)=\gamma\left(\theta_{n} *_{\alpha, \beta} \delta_{n}\right)(\lambda) \in \Delta_{E}^{(\alpha, \beta)}
$$

as $\Delta^{(\alpha, \beta)}$ is a delta sequence and $\theta_{n} *_{\alpha, \beta} \delta_{n} \in \Delta^{(\alpha, \beta)}$. The proofs of (iii)-(vi) follow from [13, Proposition 4.9] and the fact that $\beta_{1}^{(\alpha, \beta)}$ is a Boehmian space. Hence we omit the details.

The proof of this theorem is therefore finished.

For every $\left(F_{n}\right) \in L_{E}^{1}\left(\mathbb{R}, \vec{d}_{\alpha, \beta} x\right)$ and $\left(\delta_{n}^{E}\right) \in \Delta_{E}^{(\alpha, \beta)}$, the pair of sequences $\left(F_{n}, \delta_{n}^{E}\right)\left(\right.$ or $\left.\frac{F_{n}}{\delta_{n}^{E}}\right)$ is said to be a quotient of sequences in $\beta_{2}^{(\alpha, \beta)}$ if $F_{n} *_{\alpha, \beta}^{E} \delta_{m}^{E}=F_{m} *_{\alpha, \beta}^{E} \delta_{n}^{E}$, for all $n, m \in \mathbb{N}$. Hence, we may easily check that $\frac{F *_{\alpha, \beta}^{E} \beta_{n}^{E}}{\delta_{n}^{E}}, \frac{F_{n}}{\delta_{n}^{E} *_{\alpha, \beta}^{E} F}=\frac{F_{n} *_{\alpha, \beta}^{E} F}{\delta_{n}^{E}}, \frac{F_{n} *_{\alpha, \beta}^{E} \delta_{n}^{E}+G_{n} *_{\alpha, \beta}^{E} \delta_{n}^{E}}{\delta_{n}^{E} *_{\alpha, \beta} \theta_{n}^{E}} \frac{F_{n}}{\delta_{n}^{E} *_{\alpha, \beta}^{E} G_{n}}$ and $=\frac{F_{n} *_{\alpha, \beta}^{E} G_{n}}{\delta_{n}^{E}}$ when $\frac{F_{n}}{\delta_{n}^{E}}$ and $\frac{G_{n}}{\theta_{n}^{E}}$ are quotients of sequences and $F \in L_{E}^{1}\left(\mathbb{R}, \vec{d}_{\alpha, \beta} x\right)$. Moreover, the quotients $\frac{F_{n}}{\delta_{n}^{E}}$ and $\frac{G_{n}}{\theta_{n}^{E}}$ are said to be equivalent if $F_{n} *_{\alpha, \beta}^{E} \theta_{m}^{E}=G_{m} *_{\alpha, \beta}^{E} \delta_{n}^{E}$ for every $n, m \in \mathbb{N}$. The equivalent class of quotients of sequences containing $\frac{F_{n}}{\delta_{n}^{E}}$ is the Boehmian $\breve{W}=\left(\frac{F_{n}}{\delta_{n}^{E}}\right)$. The space of such Boehmians is denoted $\beta_{2}^{(\alpha, \beta)}$. For $\breve{W}=\left(\frac{F_{n}}{\delta_{n}^{E}}\right)$ and $\breve{Z}=\left(\frac{G_{n}}{\theta_{n}^{E}}\right)$ in $\beta_{2}^{(\alpha, \beta)}$, addition and scalar multiplication are, respectively, defined as $\breve{W}+\breve{Z}=\left(\frac{F_{n} * *_{\alpha, \beta}^{E} \delta_{n}^{E}+G_{n} *_{\alpha, \beta}^{E} \delta_{n}^{E}}{\delta_{n}^{E} * *_{\alpha, \beta}^{E} \beta_{n}^{E}}\right)$ and $\beta \breve{W}=\left(\frac{\beta F_{n}}{\delta_{n}^{E}}\right), \beta \in$ C. Moreover, we have $\breve{W} *_{\alpha, \beta}^{E} F=\left(\frac{F_{n} *_{\alpha, \beta}^{E} F}{\delta_{n}^{E}}\right)$ for $F \in L_{E}^{1}\left(\mathbb{R}, \vec{d}_{\alpha, \beta} x\right)$.

$\delta^{E}$-convergence: $\operatorname{In} \beta_{2}^{(\alpha, \beta)},\left(\breve{W}_{n}\right)$ is $\delta^{E}$-convergent to $\breve{W}$, denoted by $\delta^{E}-\lim _{n \rightarrow \infty} \breve{W}_{n}=\breve{W}$, provided there can be found a delta sequence $\left(\delta_{n}^{E}\right)$ such that

(i) $\left(\breve{W}_{n} *_{\alpha, \beta}^{E} \delta_{k}^{E}\right),\left(\breve{W} *_{\alpha, \beta}^{E} \delta_{k}^{E}\right)$ in $L_{E}^{1}\left(\mathbb{R}, \vec{d}_{\alpha, \beta} x\right)$, for all $n, k \in \mathbb{N}$,

(ii) $\lim _{n \rightarrow \infty} \breve{W}_{n} *_{\alpha, \beta}^{E} \delta_{k}=\breve{W} *_{\alpha, \beta} \delta_{k}$ in $L_{E}^{1}\left(\mathbb{R}, \vec{d}_{\alpha, \beta} x\right)$, for every $k \in \mathbb{N}$.

$\Delta_{E}^{(\alpha, \beta)}$-convergent: In $\beta_{2}^{(\alpha, \beta)},\left(\breve{W}_{n}\right)$ is $\Delta_{E}^{(\alpha, \beta)}$-convergent to $\breve{W}$, denoted by $\Delta_{E}^{(\alpha, \beta)}-\lim _{n \rightarrow \infty} \breve{W}_{n}=\breve{W}$, provided there can be found a delta sequence $\left(\delta_{n}^{E}\right)$ such that

(i) $\left(\breve{W}_{n}-\breve{W}\right) *_{\alpha, \beta}^{E} \delta_{n}^{E} \in L_{E}^{1}\left(\mathbb{R}, \vec{d}_{\alpha, \beta} x\right) \quad(\forall n \in \mathbb{N})$,

(ii) $\lim _{n \rightarrow \infty}\left(\breve{W}_{n}-\breve{W}\right) *_{\alpha, \beta}^{E} \delta_{n}^{E}=0 \quad$ in $L_{E}^{1}\left(\mathbb{R}, \vec{d}_{\alpha, \beta} x\right)$. 
Theorem 14 Let $\left(\Omega_{n}\right) \in \Delta_{E}^{(\alpha, \beta)}, \Omega_{n}=\gamma \psi_{n}$ for some fixed $\left(\psi_{n}\right) \in \Delta^{(\alpha, \beta)}$ and $W \in$ $L_{E}^{1}\left(\mathbb{R}, \vec{d}_{\alpha, \beta} x\right)$ where $W=\gamma \psi, \psi \in L^{1}\left(\mathbb{R}, \vec{d}_{\alpha, \beta} x\right)$, then we have the mapping

$$
W \rightarrow \breve{W}
$$

where $\breve{W}=\left(\frac{W *_{\alpha, \beta}^{E} \Omega_{n}}{\Omega_{n}}\right)$ is an injective mapping from $L_{E}^{1}\left(\mathbb{R}, \vec{d}_{\alpha, \beta} x\right)$ into $\beta_{2}^{(\alpha, \beta)}$.

Hence, from Theorem 14, it can be seen that the space $L_{E}^{1}\left(\mathbb{R}, \vec{d}_{\alpha, \beta} x\right)$ may be identified as a subspace of $\beta_{2}^{(\alpha, \beta)}$. This, indeed, leads to the following results.

Theorem 15 Let $\left(\Omega_{n}\right) \in \Delta_{E}^{(\alpha, \beta)}$. If $W_{n} \rightarrow W$ in $L_{E}^{1}\left(\mathbb{R}, \vec{d}_{\alpha, \beta} x\right)$ as $n \rightarrow \infty$, then, for all $k \in \mathbb{N}$,

$$
W_{n} *_{\alpha, \beta}^{E} \Omega_{k} \rightarrow W *_{\alpha, \beta}^{E} \Omega_{k}
$$

as $n \rightarrow \infty$. That is, $\breve{W}_{n} \rightarrow \breve{W}$ in $\beta_{2}^{(\alpha, \beta)}$ as $n \rightarrow \infty$.

Theorem 16 The mapping $W \rightarrow \breve{W}$ defined by (13) is a continuous embedding of $L_{E}^{1}\left(\mathbb{R}, \vec{d}_{\alpha, \beta} x\right)$ into the space $\beta_{2}^{(\alpha, \beta)}$.

\section{The generalized Opdam-Cherednik transform}

This section discusses a pair of generalized Opdam-Cherednik operators and derive some general properties. Based on the structure of the Boehmian spaces $\beta_{1}^{(\alpha, \beta)}$ and $\beta_{2}^{(\alpha, \beta)}$ and the convolution theorem we present the following definition.

Definition 17 The generalized Opdam-Cherednik integral operator of a Boehmian $\breve{w}$ in $\beta_{1}^{(\alpha, \beta)}$ is the Boehmian $\breve{W}$ in $\beta_{2}^{(\alpha, \beta)}$ defined by

$$
\gamma_{E} \breve{w}=\breve{W}
$$

where $\breve{w}=\left(\frac{f_{n}}{\delta_{n}}\right), f_{n} \in L^{1}\left(\mathbb{R}, \vec{d}_{\alpha, \beta} x\right), \delta_{n} \in \Delta^{(\alpha, \beta)}, \forall n \in \mathbb{N}$, and $\breve{W}=\left(\frac{\gamma f_{n}}{\gamma \delta_{n}}\right)$.

Theorem 18 Let $\breve{w}=\left(\frac{f_{n}}{\delta_{n}}\right)$ be a Boehmian in $\beta_{1}^{(\alpha, \beta)}$. Then the mapping $\breve{w} \rightarrow \breve{W}$, defined by $\breve{W}=\gamma_{E} \breve{w}$, coincides with the corresponding mapping $\gamma: L^{1}\left(\mathbb{R}, \vec{d}_{\alpha, \beta} x\right) \rightarrow L_{E}^{1}\left(\mathbb{R}, \vec{d}_{\alpha, \beta} x\right)$.

Proof Let $w \in L^{1}\left(\mathbb{R}, \vec{d}_{\alpha, \beta} x\right)$, then $\breve{w}=\left(\frac{w *_{\alpha, \beta} \delta_{n}}{\delta_{n}}\right)$ is the identification of $w$ in $\beta_{1}^{(\alpha, \beta)}$. On the other hand, (14) and [13, Proposition 4.9] reveal that the Boehmian

$$
\breve{W}=\gamma_{E} \breve{w}=\left(\frac{\gamma\left(w *_{\alpha, \beta} \delta_{n}\right)}{\gamma\left(\delta_{n}\right)}\right)=\left(\frac{W \Omega_{n}}{\Omega_{n}}\right)=\left(\frac{W *_{\alpha, \beta}^{E} \Omega_{n}}{\Omega_{n}}\right)
$$

can be identified with $W \in L_{E}^{1}\left(\mathbb{R}, \vec{d}_{\alpha, \beta} x\right)$ in $\beta_{1}^{(\alpha, \beta)}$ provided $\Omega_{n}=\gamma\left(\delta_{n}\right)$ and $W=\gamma w$.

The proof is therefore finished.

We state without proof the following characterization theorem.

Theorem 19 Let $\breve{w}=\left(\frac{f_{n}}{\delta_{n}}\right)$ and $\breve{W}=\gamma_{E} \breve{w}$. Then the mapping $\breve{w} \rightarrow \breve{W}$ is linear, bijective and continuous with respect to the convergence of the Boehmian spaces. The proof of this theorem is analogous to the proofs given in the literature (see, e.g., [22-25]). Hence, it has been omitted. 
We introduce the inverse integral operator of the operator $\gamma_{E}$ as the Boehmian in $\beta_{1}^{(\alpha, \beta)}$ defined by follows.

Definition 20 Let $\breve{W} \in \beta_{2}^{(\alpha, \beta)}$, where $\breve{W}=\gamma_{E} \breve{w}, \breve{w}=\left(\frac{f_{n}}{\delta_{n}}\right),\left(f_{n}\right) \in L^{1}\left(\mathbb{R}, \vec{d}_{\alpha, \beta} x\right)$ and $\left(\delta_{n}\right) \in$ $\Delta^{(\alpha, \beta)}$. We define the inverse $\gamma_{E}$ operator of $\breve{W}$ as

$$
\left(\gamma_{E}\right)^{-1} \breve{W}=\breve{w} .
$$

Theorem 21 The inverse operator $\breve{w} \rightarrow \breve{W}$ is linear.

Proof Let $\breve{W}$ and $\breve{X}$ be the Boehmians in $\beta_{2}^{(\alpha, \beta)}$ such that $\breve{W}=\gamma_{E} \breve{w}$ and $\breve{X}=\gamma_{E} \breve{X}$ where $\breve{w}=\left(\frac{\psi_{n}}{\delta_{n}}\right), \breve{x}=\left(\frac{x_{n}}{\epsilon_{n}}\right)$. Then, for all $n \in \mathbb{N}$ [13, Proposition 4.9], the linearity of the integral leads to

$$
\breve{W}+\breve{X}=\left(\frac{\gamma \psi_{n} *_{\alpha, \beta}^{E} \gamma \epsilon_{n}+\gamma x_{n} *_{\alpha, \beta}^{E} \gamma \epsilon_{n}}{\gamma \delta_{n} *_{\alpha, \beta}^{E} \gamma \epsilon_{n}}\right)=\left(\frac{\gamma\left(\psi_{n} *_{\alpha, \beta} \epsilon_{n}+x_{n} *_{\alpha, \beta} \delta_{n}\right)}{\gamma\left(\delta_{n} *_{\alpha, \beta} \epsilon_{n}\right)}\right) .
$$

Hence, employing Definition 20 yields

$$
\left(\gamma_{E}\right)^{-1}(\breve{W}+\breve{X})=\left(\frac{\psi_{n} *_{\alpha, \beta} \epsilon_{n}+x_{n} *_{\alpha, \beta} \delta_{n}}{\delta_{n} *_{\alpha, \beta} \epsilon_{n}}\right) .
$$

Notion of addition in $\beta_{1}^{(\alpha, \beta)}$ reveals $\left(\gamma_{E}\right)^{-1}(\breve{W}+\breve{X})=\breve{w}+\breve{x}$. To complete the proof of the theorem, we, indeed, have to mention that, for some $\eta \in \mathbb{C}$ and all $n \in \mathbb{N}$, we have

$$
\left(\gamma_{E}\right)^{-1}(\eta \breve{W})=\eta \breve{w}
$$

This finishes the proof of the theorem.

Theorem 22 Let $\breve{W} \in \beta_{2}^{(\alpha, \beta)}, \breve{W}=\gamma_{E} \breve{w}, \breve{w}=\left(\frac{\psi_{n}}{\epsilon_{n}}\right)$ and $X \in L_{E}^{1}\left(\mathbb{R}, \vec{d}_{\alpha, \beta} x\right), X=\gamma x$, $x \in L^{1}\left(\mathbb{R}, \vec{d}_{\alpha, \beta} x\right)$. Then we have $\left(\gamma_{E}\right)^{-1}\left(\breve{W} *_{\alpha, \beta}^{E} X\right)=\breve{w} *_{\alpha, \beta} x$.

Proof Assume $\breve{W} \in \beta_{2}^{(\alpha, \beta)}$ where $\breve{W}=\gamma_{E} \breve{w}, \breve{w}=\left(\frac{\psi_{n}}{\epsilon_{n}}\right)$. Hence, we have

$$
\left(\gamma_{E}\right)^{-1}\left(\breve{W} *_{\alpha, \beta}^{E} X\right)=\left(\frac{\gamma \psi_{n} *_{\alpha, \beta}^{E} \gamma x}{\digamma \epsilon_{n}}\right)
$$

Upon using [13, Proposition 4.9] and Definition 20, we obtain

$$
\left(\gamma_{E}\right)^{-1}\left(\breve{W} *_{\alpha, \beta}^{E} X\right)=\left(\gamma_{E}\right)^{-1}\left(\frac{\gamma\left(\psi_{n} *_{\alpha, \beta} x\right)}{\gamma \epsilon_{n}}\right)=\left(\frac{\psi_{n}}{\epsilon_{n}} *_{\alpha, \beta} x\right)=\breve{w} *_{\alpha, \beta} x .
$$

The proof of this theorem is, therefore, finished.

Theorem 23 Let $\breve{W} \in \beta_{2}^{(\alpha, \beta)}, \breve{W}=\gamma_{E} \breve{w}, \breve{w}=\left(\frac{\psi_{n}}{\delta_{n}}\right)$ and $X \in L_{E}^{1}\left(\mathbb{R}, \vec{d}_{\alpha, \beta} x\right), X=\gamma x, x \in$ $L^{1}\left(\mathbb{R}, \vec{d}_{\alpha, \beta} x\right)$. Then we have $\gamma_{E}\left(\breve{w} *_{\alpha, \beta} x\right)=\breve{W} *_{\alpha, \beta}^{E} X$.

The proof of this theorem is analogous to the proof of Theorem 22. Details are, therefore, omitted. 
Theorem 24 Let $\breve{W} \in \beta_{2}^{(\alpha, \beta)}, \breve{W}=\gamma_{E} \breve{w}, \breve{w}=\left(\frac{f_{n}}{\delta_{n}}\right)$, then $\breve{W}$ is in the range of $\gamma_{E}$ iff $\gamma f_{n}$ is in the range of $\gamma$, for $\left(f_{n}\right) \in L^{1}\left(\mathbb{R}, \vec{d}_{\alpha, \beta} x\right)$ and $\left(\delta_{n}\right) \in \Delta^{(\alpha, \beta)}$.

Proof Let $\breve{W}$ be in the range of $\gamma_{E}, \breve{W}=\gamma_{E} \breve{w}, \breve{w}=\left(\frac{f_{n}}{\delta_{n}}\right), f_{n} \in L^{1}\left(\mathbb{R}, \vec{d}_{\alpha, \beta} x\right)$ and $\delta_{n} \in \Delta^{(\alpha, \beta)}$, for all $n \in \mathbb{N}$. Then it is clear that $f_{n}$ is in the range of $\gamma$ for all $n \in \mathbb{N}$. On the other hand, if $\breve{W}$ is in the range of $\gamma_{E}$, then $\breve{W}=\gamma_{E} \breve{w}, \breve{w}=\left(\frac{f_{n}}{\delta_{n}}\right), f_{n} \in L^{1}\left(\mathbb{R}, \vec{d}_{\alpha, \beta} x\right), \delta_{n} \in \Delta^{(\alpha, \beta)}, \forall n \in \mathbb{N}$. Therefore, the equivalence relation in $\beta_{2}^{(\alpha, \beta)}$ leads to

$$
\gamma f_{n} *_{\alpha, \beta}^{E} \gamma \delta_{m}=\gamma f_{m} *_{\alpha, \beta}^{E} \gamma \delta_{n}
$$

Therefore, [13, Proposition 4.9] states that $\gamma\left(f_{n} *_{\alpha, \beta} \delta_{m}\right)=\gamma\left(f_{m} *_{\alpha, \beta} \delta_{n}\right)$. Hence, it follows that $f_{n} *_{\alpha, \beta} \delta_{m}=f_{m} *_{\alpha, \beta} \delta_{n}$. That is, $\frac{f_{n}}{\delta_{n}}$ is a quotient of sequences and the equivalence class $\left(\frac{f_{n}}{\delta_{n}}\right)=\breve{w}$ containing $\frac{f_{n}}{\delta_{n}}$ is the Boehmian in $\beta_{1}^{(\alpha, \beta)}$ satisfying $\gamma_{E} \breve{W}=\breve{W}$.

This finishes the proof of the theorem.

\section{Conclusion}

This paper has demonstrated the possibility of extending the Opdam-Cherednik integral operator into a class of generalized functions. It proposes sets of delta sequences and convolution products and investigates the classes of Boehmians. Moreover, this paper considers a generalization of the Opdam-Cherednik integral operator and its inversion formula on the constructed spaces of Boehmians. Furthermore, it derives various properties of the new pair of generalized Opdam-Cherednik integral operators in a generalized context.

Acknowledgements

The authors would like to thank the referees for their insightful comments and Springer Nature for its support.

Funding

No funding sources to be declared.

Availability of data and materials

Please contact author for data requests.

Competing interests

The authors declare that they have no competing interests.

Authors' contributions

The authors contributed equally and significantly in writing this paper. All authors read and approved the final manuscript.

\section{Author details}

'Department of Physics and Basic Sciences, Faculty of Engineering Technology, Al-Balqa Applied University, 11134 , Amman, Jordan. ${ }^{2}$ Department of Economics, Faculty of Economics, Administrative and Social Sciences, Hasan Kalyoncu University, TR-27410, Gaziantep, Turkey. ${ }^{3}$ Department of Applied Science, Ajloun College, Al-Balqa Applied University, Al-Salt, Jordan. ${ }^{4}$ Nonlinear Dynamics Research Center (NDRC), Ajman University, Ajman, UAE.

\section{Publisher's Note}

Springer Nature remains neutral with regard to jurisdictional claims in published maps and institutional affiliations.

Received: 16 May 2021 Accepted: 29 June 2021 Published online: 16 July 2021

\section{References}

1. Mikusinski, P.: Fourier transform for integrable Boehmians. Rocky Mt. J. Math. 17(3), 577-582 (1987)

2. Al-Omari, S., Baleanu, D.: On the generalized Stieltjes transform of Fox's kernel function and its properties in the space of generalized functions. J. Comput. Anal. Appl. 23(1), 108-118 (2017)

3. Nemzer, D:: The Laplace transform on a class of Boehmians. Bull. Aust. Math. Soc. 46, 347-352 (1992)

4. Bhuvaneswari, R., Karunakaran, V.: Boehmians of type $S$ and their Fourier transforms. Annales UMCS, Math. 64, 27-43 (2010) 
5. Nemzer, D.: One-parameter groups of Boehmians. Bull. Korean Math. Soc. 44, 419-428 (2007)

6. Karunakaran, V., Kalpakam, N.V.: Hilbert transform for Boehmians. Integral Transforms Spec. Funct. 9(1), 19-36 (2000)

7. Nemzer, D.: Periodic Boehmians. Int. J. Math. Math. Sci. 12, 685-692 (1989)

8. Al-Omari, S., Park, J., Agarwal, P.: An extension of some variant of Meijer type integrals in the class of Boehmian. J. Inequal. Appl. 2016, 1 (2016)

9. Al-Omari, S., Baleanu, D.: A quadratic-phase integral operator for sets of generalized integrable functions. Math. Methods Appl. Sci. 43(7), 4168-4176 (2020)

10. Al-Omari, S.: Some remarks on short-time Fourier integral operators and classes of rapidly decaying functions. Math. Methods Appl. Sci. 41, 1-8 (2018)

11. El Ouadih, S., Daher, R.: Some new estimates for the Cherednik-Opdam transform in the space $L_{\alpha, \beta}^{2}(R)$. J. Pseudo-Differ. Oper. Appl. 7, 47-58 (2016)

12. Schapira, B:: Cntributions to the hypergeometric function theory of the Heckman and Opdam: Sharp estimates, Schwartz space, heat kernel, GAFA. Geom. Funct. Anal. 18, 222-250 (2008)

13. Anker, J.P., Ayadi, F., Sifi, M.: Opdam's hypergeometric functions: product formula and convolution structure in dimension 1. Adv. Pure Appl. Math. 3, 11-44 (2012)

14. Mejjaoli, H.: Qualitative uncertainty principles for the Opdam-Cherednik transform. Integral Transforms Spec. Funct. 25(7), 528-546 (2014)

15. Mejjaoli, $\mathrm{H} .:$ On the range of the Opdam-Cherednik transform and Roe's theorem in the Cherednik setting. J. Pseudo-Differ. Oper. Appl. 7(1), 19-45 (2016)

16. Achak, A., Daher, R.: Benedicks-Amrein-Berthier type theorem related to Opdam-Cherednik transform. J. Pseudo-Differ. Oper. Appl. 9(2), 431-441 (2018)

17. Shimeno, N.: An analogue of Hardy's theorem for the Heckman-Opdam transform. J. Math. Kyoto Univ. 41(2), 251-256 (2001)

18. El Ouadih, S., Daher, R.: Titmarsh's theorem for the Cherednik-Opdam transform in the space $L^{2}(R)$. Int. J. Anal. Appl. 9(2), 90-95 (2015)

19. Mejjaoli, H.: On the range of the Opdam-Cherednik transform and Roe's theorem in the Cherednik setting. J. Pseudo-Differ. Oper. Appl. 7(1), 19-45 (2015)

20. El Ouadih, S., Daher, R.: Growth properties of the Cherednik-Opdam transform in the space $L^{p}$. Casp. J. Math. Sci. 7(1), 80-87 (2018)

21. Ben Salem, N., Salem, O.A.: Convolution structure associated with the Jacobi-Dunkl operator on $\mathbb{R}$. Ramanujan J. 12, 359-378 (2006)

22. Al-Omari, S., Baleanu, D.: Some remarks on short-time Fourier integral operators and classes of rapidly decaying functions. Math. Methods Appl. Sci. 42(16), 1-8 (2019)

23. Al-Omari, S., Baleanu, D.: Quaternion Fourier integral operators for spaces of generalized quaternions. Math. Methods Appl. Sci. 41(18), 1-8 (2018)

24. Al-Omari, S.: Some estimate of a generalized Bessel-Struve transform on certain space of generalized functions. Ukr. Math. J. 69(9), 1155-1165 (2017)

25. Al-Omari, S.: Some characteristics of S transforms in a class of rapidly decreasing Boehmians. J. Pseudo-Differ. Oper. Appl. 5(4), 527-537 (2014)

\section{Submit your manuscript to a SpringerOpen ${ }^{\circ}$ journal and benefit from:}

- Convenient online submission

- Rigorous peer review

- Open access: articles freely available online

- High visibility within the field

- Retaining the copyright to your article

Submit your next manuscript at springeropen.com 